\title{
A NOTE ON PRIMITIVE SKEW CURVES
}

\author{
DICK WICK HALL
}

In 1930 Kuratowski ${ }^{1}$ established the following result:

TheOREM A. A locally connected continuum, containing but a finite number of simple closed curves, is homeomorphic with a subset of the plane, provided that it does not contain a primitive skew curve of type I or a primitive skew curve of type II.

By a primitive skew curve of type I we mean any topological image of the complex $C$ which consists of two groups of three vertices each and nine 1-cells, in a fashion that each vertex of one group together with each vertex of the other group bounds a 1-cell. By a primitive skew curve of type II we mean any topological image of the complex $D$ which consists of five vertices and ten 1-cells in a fashion that each pair of vertices bounds a 1 -cell.

In 1934 Claytor $^{2}$ proved that every cyclic locally connected continuum containing no primitive skew curve of either type must be homeomorphic with a subset of a spherical surface.

In this note we point out that for a large class of locally connected continua the property of being planar may be insured merely by requiring that the given locally connected continuum contain no primitive skew curve of type I. Stated precisely, our principal theorem is the following:

THEOREM 1. Let $M$ be a locally connected continuum separated by no pair of its points and assume that $M$ contains no primitive skew curve of type I. Then $M$ contains no primitive skew curve of type II.

The proof is immediate. Assuming the existence of a primitive skew curve $D$ of type II in $M$ and using the fact that no two points separate $M$ we may easily find a simple arc $a b$ in $M$ having only the points $a$ and $b$ in common with $D$ and such that $a$ is interior to a free arc of $D$ while $b$ does not lie on the closure of this free arc. Denote the sum of $D$ and the arc $a b$ by $D^{\prime}$. A careful examination of $D^{\prime}$ shows that this set must contain a primitive skew curve of type I contrary to our hypothesis on $M$.

\footnotetext{
Presented to the Society, September 13, 1943; received by the editors July 17, 1943.

${ }^{1}$ Sur les problème des courbes gauches en topologie, Fund. Math. vol. 15 (1930) pp. 271-283.

2 Topological immersion of Peanian continua in a spherical surface, Ann. of Math. vol. 35 (1934) pp. 809-835.
} 
It is natural to inquire whether or not the above result remains true when the types of primitive skew curves there mentioned are interchanged. To show that this is not the case we need only construct a locally connected continuum $K$ which is separated by no pair of its points, but such that $K$ contains a primitive skew curve of type I but no primitive skew curve of type II. To do this let $K$ consist of three 2-cells $E, F, G$ having their interiors disjoint and exactly three boundary points $a, b, c$ in common. It is self-evident that no two points separate $K$ while $K$ contains a primitive skew curve of type I. It is easy to show that $K$ contains no primitive skew curve of type II by making use of the fact that no two vertices of such a primitive skew curve can be separared by the removal of any three points of the curve.

As an application of our theorem we prove the following unpublished theorem of F. B. Jones: ${ }^{3}$

THEOREM 2 (JoNes). Let $M$ be a locally connected continuum separated by no pair of its points but by every one of its simple closed curves, and suppose that $M$ contains no primitive skew curve of type I. Then $M$ is homeomorphic with a spherical surface.

Proof. By Theorem 1 and the theorem of Claytor cited above we know that $M$ is homeomorphic to a subset of a spherical surface $S$. If the theorem is false then $M$ has a complementary domain $D$ in $S$. By a theorem of R. L. Moore the boundary of $D$ is a simple closed curve $J$. Now $J$ must separate $M$. Using this fact together with the knowledge that every component of $M-J$ must lie on the side of $J$ opposite to $D$ we easily see that some pair of points of $M$ must separate $M$. This contradiction completes the proof of the theorem.

UNIVERSITY OF MARYLAND

${ }^{3}$ See Bull. Amer. Math. Soc. abstract 48-11-340.

${ }^{4}$ Concerning the common boundary of two domains, Fund. Math. vol. 6 (1924) pp. 203-213. 